\title{
COMPARISON OF DISCRETE-TIME APPROXIMATIONS FOR CONTINUOUS-TIME NONLINEAR SYSTEMS
}

\author{
H. Koeppl \\ Christian Doppler Laboratory \\ for Nonlinear Signal Processing \\ Graz University of Technology, Austria \\ heinz.koeppl@tugraz.at
}

\author{
D. Schwingshackl
}

\begin{abstract}
The work addresses the problem of approximating the sampled input-output (i/o) behavior of continuous-time nonlinear systems using discrete-time Volterra models. For an exactly band-limited nonlinear system for which a Volterra representation exists, the discrete-time Volterra model exactly corresponds to the sampled continuous-time Volterra kernels. Physical systems, as they are causal, are never exactly band-limited. Thus, a modeling error is introduced. By relaxing the causality condition and allowing a small processing delay, it is shown through simulation that more accurate discrete-time Volterra models compared to sampled continuous-time Volterra models can be generated.
\end{abstract}

\section{INTRODUCTION}

The subject of this work is to model the sampled i/o characteristics of continuous-time nonlinear systems. The model should be valid for nonlinear systems which have fading memory [1] and therefore can be represented by Volterra systems. A block diagram of the modeling setup is depicted in Fig. 1, where $\mathrm{V}$ denotes the i/o operator of the nonlinear system with $y(t)=(\mathrm{V} u)(t)$, and $\mathrm{V}^{\mathrm{d}}$ denotes the $\mathrm{i} / \mathrm{o}$ operator of the discrete-time approximation, with $\bar{y}[n]=\left(\mathrm{V}^{\mathrm{d}} \bar{u}\right)[n]$. This situation could correspond to mixed signal circuit anal-

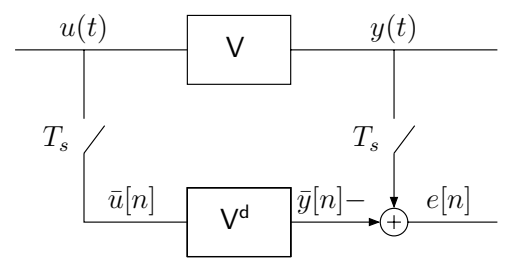

Fig. 1. Setup for the discussed identification problem; continuous-time system $\mathrm{V}$ and discrete-time system $\mathrm{V}^{\mathrm{d}}$.

\author{
Infineon Technologies, \\ Design Center Villach, Austria and \\ Christian Doppler Laboratory \\ for Nonlinear Signal Processing \\ Graz University of Technology, Austria \\ schwingshackl@ieee.org
}

ysis and design, where a discrete-time model of an analog circuit is generated to be implemented either in the digital circuit part or in some digital circuit simulator. One application would be a digital predistortion of an analog circuit. Two conditions, which are often met in these scenarios, are that the sampling frequency of the digital part is fixed through the circuit design and that analog circuits are in general only weakly nonlinear systems. These conditions imply that the sampling frequency cannot be chosen arbitrarily and that a Volterra series expansion is a feasible approach to model these systems. If the model is generated by sampling the $p$-th order continuous-time Volterra kernels $h_{p}(\boldsymbol{\tau})$, with $\boldsymbol{\tau} \equiv\left[\tau_{1}, \ldots, \tau_{p}\right]^{T}$ of the system $\mathrm{V}$, at the predetermined sampling rate $F_{s}$, the model $\mathrm{V}^{\mathrm{d}}$ will not be accurate in general. That is because the frequency domain kernels $H_{p}(j \boldsymbol{\Omega})$, with $\boldsymbol{\Omega} \equiv\left[\Omega_{1}, \ldots, \Omega_{p}\right]^{T}$, of $\mathrm{V}$ do not obey $H_{p}(j \boldsymbol{\Omega})=0$, if any $\left|\Omega_{m}\right|>\pi F_{s}, m=1, \ldots, p$, which is required to achieve an exact model. For a physical system $\checkmark$ no such $F_{s}$ can be found to yield an exact model because system $\mathrm{V}$ is causal and thus cannot be strictly band-limited.

Subsequently, based on [2] a method that takes into account the problem of not band-limited nonlinear systems is presented and evaluated. The method yields non-causal discrete-time models, thus for realtime applications the induced processing delay $\delta$ has to be considered. That method is referred to as "non-causal system method" in the following. The accuracy of the model is compared with the standard method of sampling the kernels $h_{p}(\boldsymbol{\tau})$ of $\mathrm{V}$ with $F_{s}=1 / T_{s}$ which is denoted as "impulse invariance method" in analogy to the linear filter case. For the nonlinear continuous-time reference system $\mathrm{V}$ different single-input-single-output (SISO) bilinear systems of the form

$$
\begin{aligned}
\dot{\mathbf{x}} & =\mathbf{A} \mathbf{x}+\mathbf{N} \mathbf{x} u+\mathbf{b} u \\
y & =\mathbf{c}^{T} \mathbf{x}
\end{aligned}
$$


are considered. The class of bilinear system is chosen because of their compact time and frequency Volterra representation (cf. to (7) and (8),(9) below) as well as their ability to model a broad class of nonlinear systems via the method of Carleman linearization [3, p.105]. The performance measure taken for the comparison of the two methods is the mean square error (MSE) $\epsilon_{m s}$ between the sampled output $y\left(n T_{s}\right)$ of the continuous-time system $\vee$ and the output $\bar{y}[n]$ of the discrete-time approximation $\mathrm{V}^{\mathrm{d}}$.

$$
\epsilon_{m s}=\frac{1}{\max _{n} y\left(n T_{s}\right)^{2} N_{s}} \sum_{n=1}^{N_{s}}\left(y\left(n T_{s}\right)-\bar{y}[n]\right)^{2},
$$

where the error is normalized by $\max _{n} y\left(n T_{s}\right)^{2}$. The evaluation is done using a broadband discrete-multitone (DMT) signal

$$
u(t)=\sum_{m=1}^{M_{c}} A_{m} \sin \left(m \Omega_{0} t+\varphi_{m}\right)
$$

with $M_{c}$ carriers and frequency spacing $\Omega_{0}$. A DMT signal is used because of its ability to persistently excite all modes of the nonlinear system and because of its relevance in ADSL data-transmission systems [4].

The work proceeds as follows. In section 2 the two discrete-time approximation methods are introduced. The section 3 discusses performance results of these methods by applying them to the approximation of continuous-time bilinear systems, while section 4 draws conclusions and gives an outlook.

\section{DISCRETE-TIME MODELS}

\subsection{Impulse invariance method}

The generalization of the impulse invariance method of linear filters [5, p.106] yields the discrete-time kernel $h_{p}^{d}[\mathbf{n}]$ by sampling the continuous-time Volterra kernel $h_{p}(\boldsymbol{\tau})$

$$
h_{p}^{d}[\mathbf{n}]=\left(T_{s}\right)^{p} h_{p}\left(\mathbf{n} T_{s}\right) \quad \text { with } \quad \mathbf{n} \equiv\left[n_{1}, \ldots, n_{p}\right]^{T} .
$$

For not strictly band-limited kernel functions $h_{p}(\boldsymbol{\tau})$ the sampling process (4) introduces aliasing effects and thus the continuous-frequency domain kernel of the discretetime approximation (4)

$H_{p}^{d}(j \boldsymbol{\omega})=\sum_{\mathbf{n}=-\infty}^{\infty} h_{p}^{d}[\mathbf{n}] e^{-j \boldsymbol{\omega}^{T} \mathbf{n}} \quad$ with $\quad \boldsymbol{\omega} \equiv\left[\omega_{1}, \ldots, \omega_{p}\right]^{T}$

evaluated at $\boldsymbol{\omega}=\boldsymbol{\Omega} T_{s}$ do not coincide with the frequency domain kernel $H_{p}(j \boldsymbol{\Omega})$ of the continuous-time system. Where the notation is used, that a vector valued summation index with $p$ elements refers to a $p$ fold summation.

\subsection{Non-causal system method}

The non-causal system method guarantees by its construction that the continuous-frequency domain transforms $H_{p}(j \boldsymbol{\Omega})$ and $H_{p}^{d}(j \boldsymbol{\omega})$ of the continuous-time kernel and the discrete-time kernels, respectively, coincide in the frequency band of interest, i.e. $\left[-\Omega_{s} / 2, \Omega_{s} / 2\right]^{p}$ and $[-\pi, \pi]^{p}$, respectively. This is realized through a multiplication of $H_{p}(\boldsymbol{\Omega})$ with a $p$-dimensional rectangular window function $W_{p}(j \boldsymbol{\Omega})=W\left(j \Omega_{1}\right) \cdots W\left(j \Omega_{p}\right)$

$$
W(j \Omega)= \begin{cases}1 & \text { if }|\Omega|<\pi F_{s} \\ 0 & \text { otherwise }\end{cases}
$$

with the time-domain representation

$$
w(\tau)=F_{s} \frac{\sin \left(\pi F_{s} \tau\right)}{\pi F_{s} \tau}=F_{s} \operatorname{sinc}\left(\pi F_{s} \tau\right)
$$

The multiplication yields,

$$
\tilde{H}_{p}(j \boldsymbol{\Omega})=H_{p}(j \boldsymbol{\Omega}) W_{p}(j \boldsymbol{\Omega})
$$

Thus, the discrete-time kernels of the non-causal system method are the samples of

$$
\tilde{h}_{p}\left(\mathbf{n} T_{s}\right)=\int_{\mathbb{R}^{p}} w\left(\tau_{1}\right) \cdots w\left(\tau_{p}\right) h_{p}\left(\mathbf{n} T_{s}-\boldsymbol{\tau}\right) \mathrm{d} \boldsymbol{\tau},
$$

that is

$$
\tilde{h}_{p}^{d}[\mathbf{n}]=\left(T_{s}\right)^{p} \tilde{h}_{p}\left(\mathbf{n} T_{s}\right) .
$$

An approximation $h_{p}^{d c}[\mathbf{n}]$ to (6), which can be implemented efficiently, is that $\tilde{H}_{p}(j \boldsymbol{\Omega})$ is sampled

$\tilde{H}_{p}^{d c}[\mathbf{k}]=\tilde{H}_{p}\left(j \Omega_{s}(-1 / 2+\mathbf{k} / N)\right) \quad$ with $\quad \mathbf{k} \equiv\left[k_{1}, \ldots, k_{p}\right]^{T}$

and an inverse discrete Fourier transform of $\tilde{H}_{p}^{d c}[\mathbf{k}]$,

$$
h_{p}^{d c}[\mathbf{n}]=\sum_{\mathbf{k}=0}^{N-1} \tilde{H}_{p}^{d c}[\mathbf{k}] e^{j(2 \pi / N) \mathbf{k}^{T} \mathbf{n}},
$$

is performed. This approximation corresponds to replacing the linear convolution of (5) by the circular convolution. For kernel functions $h_{p}^{d c}[\mathbf{n}]$ that decay rapidly to zero in the sample interval $[0, N-1]^{p}$ the aliasing due to the circular convolution can be negligible small. Finally, to generate a causal approximation to (1) the circular shift $\delta$ has to be performed so that the new kernel indices are $\mathbf{n}^{\prime}=$ $(\mathbf{n}-\delta) \bmod N$. An automatic determination of the size of the required circular shift $\delta$ can be done by finding the minimum of the smoothed absolute values of the "diagonal" elements of the kernel $h_{p}^{d c}[\mathbf{n}]$, i.e.

$$
S_{p}[n]=\frac{1}{2 M+1} \sum_{m=-M}^{M}\left|h_{p}^{d c}[\mathbf{i}(n-m) \bmod (N-1)]\right|,
$$




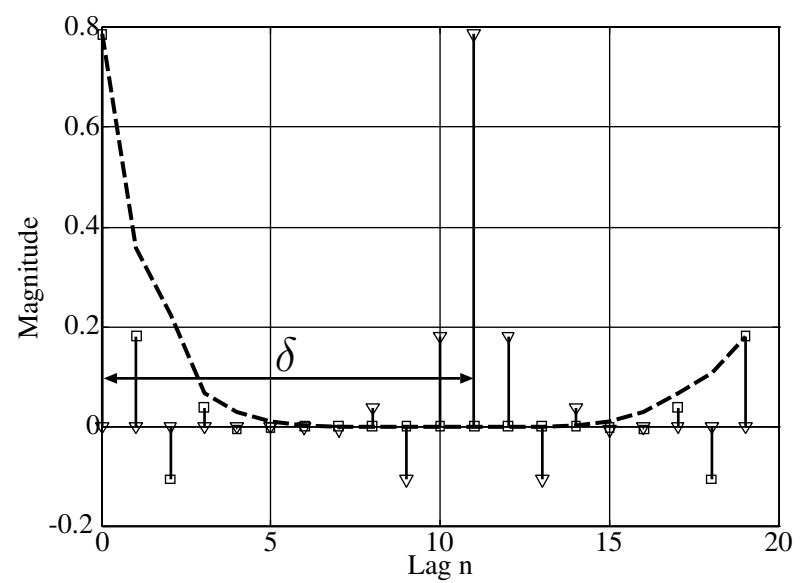

Fig. 2. Illustration of the circular shift $\delta$ and its determination via $S_{p}[n]$ (dashed) for a first order Volterra kernel; inverse discrete Fourier transform $h_{1}^{d c}[n]$ (square); shifted version $h_{1}^{d c}\left[n^{\prime}\right]$ (triangle).

i.e., $\delta=\arg \min _{n}\left(S_{p}[n]\right)$, with the $p$-element vector $\mathbf{i} \equiv$ $[1, \ldots, 1]^{T}$. The meaning of the circular shift $\delta$ and that of $S_{p}[n]$ is illustrated in Fig. 2. From the viewpoint of processing delay the determined shift $\delta$ is not optimal. The proposed scheme for generating a discrete-time equivalent to a continuous-time Volterra system is summarized in the block diagram of Fig. 3.

\section{APPLICATION TO BILINEAR SYSTEMS}

In the following the methods described in section 2 are applied to the approximation of bilinear systems (1). The comparison is done based on the MSE of (2) between the output of respective discrete-time model and the sampled output of the continuous-time reference system, i.e. system (1). A DMT signal (3) comprising $M_{c}=128$ carriers equally spread over the entire bandwidth, i.e. $\left[\Omega_{0}, \Omega_{s} / 2-\Omega_{0}\right]$ is used for evaluation.

The time and frequency domain expressions for the kernels of a bilinear system (1) can be found in [6, p.106]. For the following simulation only the first and second order kernels are used. The expressions in the time-domain read

$$
\begin{aligned}
h_{1}(\tau) & =\mathbf{c}^{T} e^{\mathbf{A} \tau} \mathbf{b}, \\
h_{2}\left(\tau_{1}, \tau_{2}\right) & =\mathbf{c}^{T} e^{\mathbf{A} \tau_{1}} \mathbf{N} e^{\mathbf{A}\left(\tau_{2}-\tau_{1}\right)} \mathbf{b}
\end{aligned}
$$

and in the frequency domain

$$
H_{1}(j \Omega)=\mathbf{c}^{T}(j \Omega \mathbf{I}-\mathbf{A})^{-1} \mathbf{b},
$$

$$
\begin{aligned}
& H_{2}\left(j \Omega_{1}, j \Omega_{2}\right) \\
& =\mathbf{c}^{T}\left(\mathbf{I}\left(j \Omega_{1}+j \Omega_{2}\right)-\mathbf{A}\right)^{-1} \mathbf{N}\left(\mathbf{I} j \Omega_{1}-\mathbf{A}\right)^{-1} \mathbf{b} .
\end{aligned}
$$

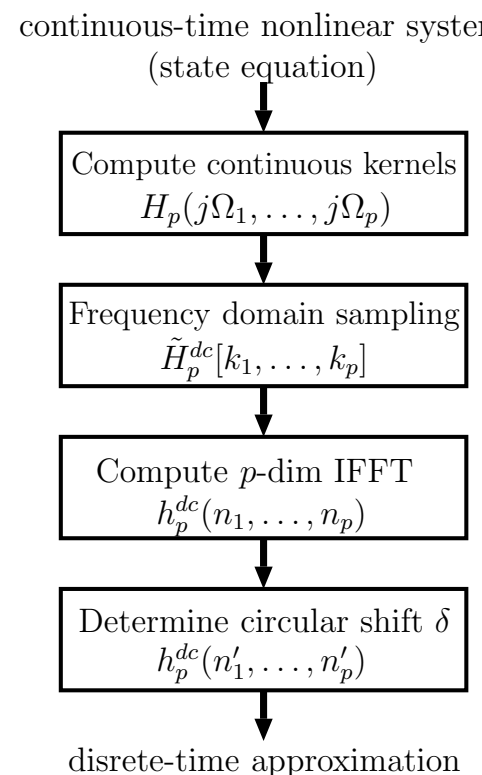

(Volterra)

Fig. 3. Block diagram of proposed procedure to compute discrete-time Volterra approximation to a continuous-time Volterra system.

\subsection{Two-dimensional bilinear system}

In the following, different bilinear systems of the form

$$
\begin{gathered}
\mathbf{A}=\left[\begin{array}{cc}
-\alpha_{1} & -\alpha_{2} \\
1 & 0
\end{array}\right] \quad \text { with } \quad \mathbf{N}=\left[\begin{array}{cc}
0 & N_{12} \\
0 & 0
\end{array}\right] \\
\mathbf{b}=\left[\begin{array}{ll}
1 & 0
\end{array}\right]^{T} \text { and } \mathbf{c}=\left[\begin{array}{ll}
\beta_{1} & \beta_{2}
\end{array}\right]^{T},
\end{gathered}
$$

are generated. The Volterra kernels $H_{p}(j \Omega)$ of all systems (10) possess low pass characteristics. They differ in their attenuation at $\Omega=\mathbf{i} \Omega_{s} / 2$ and thus they represent stronger or lesser band-limited nonlinear systems. The design of the systems is performed as follows. In (10) $\mathbf{A}, \mathbf{b}$ and $\mathbf{c}$ are given in the controllable canonical form for linear systems, in which $\alpha_{i}$ and $\beta_{i}$ are the coefficients of the numerator and the denominator polynomial for a linear system. The coefficients of $\alpha_{i}$ and $\beta_{i}$ are chosen in such a way that the linear frequency response of (10) shows different cutoff frequencies $f_{c}$. A Chebychev type I low pass filter design is applied to determine the coefficients of $\alpha_{i}$ and $\beta_{i}$ for these cutoff frequencies. To approximate the sampled i/o-characteristics of (10) a second order Volterra system is considered. Applying (8) the different frequency responses $H_{1}(j \Omega)$ are shown in Fig. 4. Using (9) the effect on the second order kernels $H_{2}\left(j \Omega_{1}, j \Omega_{2}\right)$ is given in Fig. 5, which shows only its diagonal elements with $\Omega_{1}=\Omega_{2}$. This graphical representation was chosen because it gives a more quantitative information than a two-dimensional plot which shows the entire $H_{2}\left(j \Omega_{1}, j \Omega_{2}\right)$. Evaluating the performance of the 


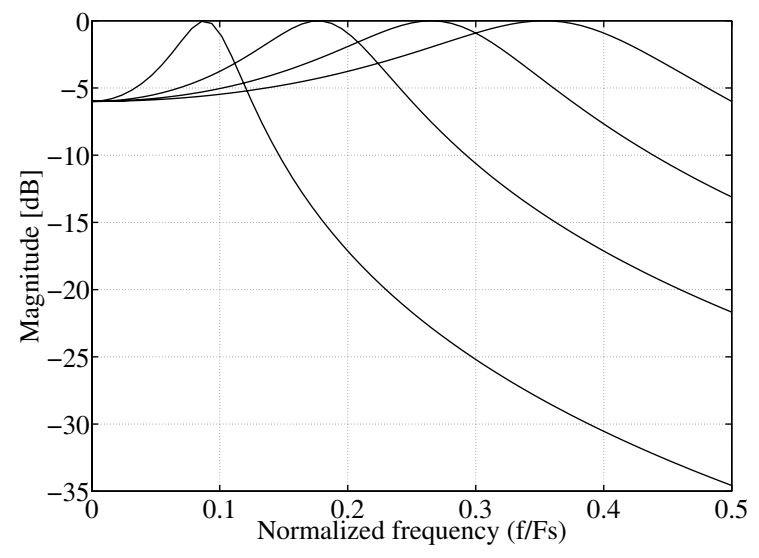

Fig. 4. Absolute values of the frequency responses $H_{1}(j \Omega)$ of the linear subsystem of (10) for different cutoff frequencies $f_{c}$.

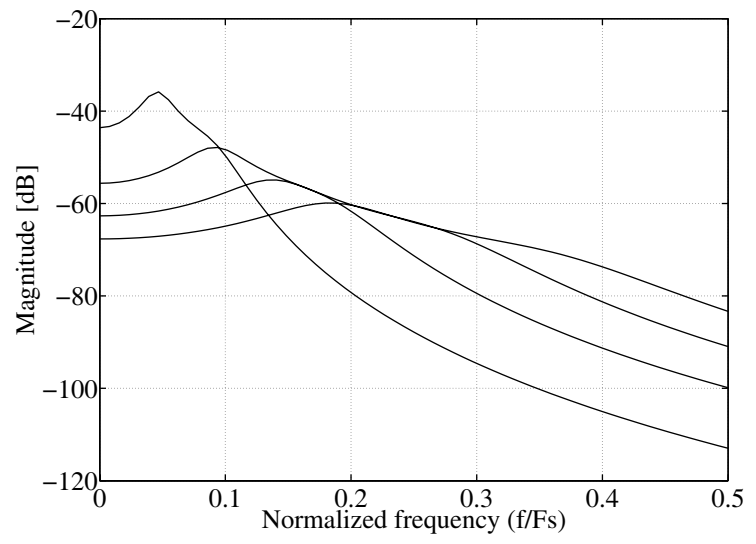

Fig. 5. Absolute values of the second order frequencydomain Volterra kernels $H_{2}\left(j \Omega_{1}, j \Omega_{2}\right)$ evaluated at $\Omega_{1}=$ $\Omega_{2}$ for different cutoff frequencies $f_{c}$ of the linear subsystem of (10).

discrete-time models generated by the methods in section 2 using (2), results in Fig. 6. It clearly shows that the proposed method outperforms the method based on the impulse invariance principle.

\section{CONCLUSION}

The scheme for generating discrete-time Volterra models of continuous-time nonlinear systems in [2] is evaluated and extended. The method shows superior performance than the method which just samples the continuous-time Volterra kernels, but introduces a processing delay. A trade-off between processing delay necessary to implement the derived discrete-time model and the accuracy of the model could be, to apply other windowing functions $W(j \Omega)$ that speeds up the decrease of the non-causal part of the kernel functions

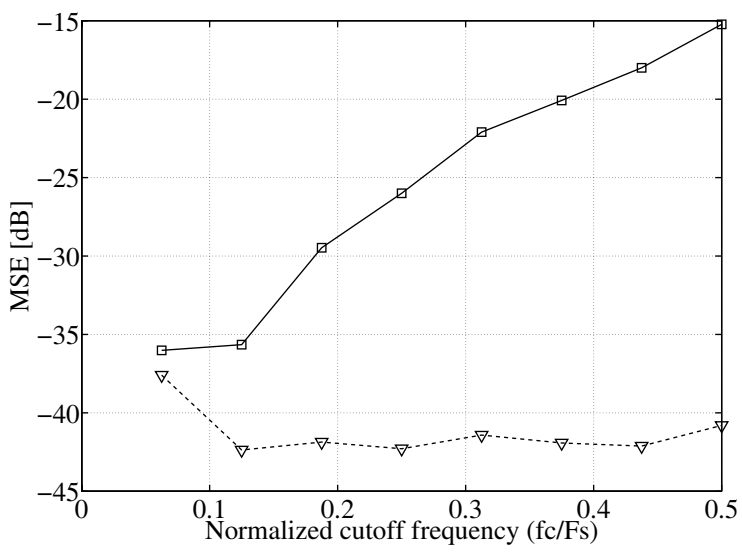

Fig. 6. Mean square error $\epsilon_{m s}$ of the impulse invariance method (solid) and the non-causal system method (dashed) for different cutoff frequencies $f_{c}$ of the linear subsystem of the bilinear example (10).

(6).

\section{REFERENCES}

[1] S. Boyd and L. O. Chua, "Fading memory and the problem of approximating nonlinear operators with Volterra series," IEEE Transactions on Circuits and Systems, vol. 32, no. 11, pp. 1150-1171, November 1985.

[2] D. Schwingshackl, G. Kubin, and G. Paoli, "Causality considerations for the identification of continoustime Volterra system," in Proceedings IEEE Workshop on Nonlinear Dynamics of Electronic Systems (NDES), 2003, pp. 241-244.

[3] W. J. Rugh, Nonlinear System Theory, Johns Hopkins University Press, London, 1981.

[4] H. Koeppl and G. Paoli, "Nonlinear system identification of a broadband subscriber line interface circuitry using the Volterra approach," in Mathematics of Signal Processing V, J. G. McWhirter and I. K. Proudler, Eds., chapter 13. Oxford University Press, 2002.

[5] A. V. Oppenheim, R. W. Schafer, and J. R. Buck, Discrete-Time Signal Processing, Prentice Hall, 1999.

[6] R. R. Mohler, Nonlinear Systems, vol. II Application to bilinear control, Prentice-Hall, Englewood Cliffs, New Jersey, 1991. 